\title{
Output-only structural damage detection based on transmissibility measurements and kernel principal component analysis
}

\author{
A. Santos, M. F. M. Silva, R. Santos, E. Figueiredo, N. M. M. Maia, and J. C. W. A. Costa, Member, IEEE
}

\begin{abstract}
Frequency response functions have been employed as damage-sensitive features in the vibration-based structural damage detection. However, the need for measuring the excitation forces arises as a remarkable limitation on the application of those features in real-world applications. As an alternative, transmissibility measurements can be explored as features with output-only nature, which implies the need for measuring only the response signals. In this paper, an output-only damage detection method is proposed, combining transmissibilities with kernel principal component analysis (KPCA). This technique is based on the pattern recognition paradigm for structural health monitoring, where feature extraction and feature classification phases are considered. In the first phase, the dimensionality of the transmissibilities is appropriately reduced by applying the KPCA algorithm. In the second phase, an outlier detection strategy is used to determine the condition of the instrumented structure. The possibility of clustering in the high-dimensional space mapped by KPCA is also reported and discussed. The proposed method is experimentally validated with transmissibilities acquired, under distinct structural conditions, from a laboratory steel beam instrumented with several accelerometers. The results demonstrate that the output-only method has high potential to be applied in a wide range of monitoring solutions, where economic issues and life-safety are primary motivations.
\end{abstract}

Index Terms-Structural health monitoring, Transmissibility measurements, Damage detection, Kernel principal component analysis.

\section{INTRODUCTION}

$\mathbf{I}$ $\mathrm{N}$ the structural health monitoring (SHM) field, damage detection based on the vibration response measurements from engineering structures has become a crucial research area due to its potential to be applied in real-world scenarios [2], [3]. Assuming that the vibration signals are often available and

This work was supported in part by the National Council for Scientific and Technological Development, under Grant 432668/2018-7 and in part by the Coordination for the Improvement of Higher Education Personnel, Brazil.

A. Santos is with Faculty of Computing and Electrical Engineering, Universidade Federal do Sul e Sudeste do Pará, F. 17, Q. 4, L. E., Marabá, Pará 68505-080, Brazil (e-mail: adamdreyton@unifesspa.edu.br).

M. F. M. Silva, R. Santos, and J. C. W. A. Costa are with Applied Electromagnetism Laboratory, Federal University of Pará, R. Augusto Corrêa 01, Belém, Pará 66075-110, Brazil.

E. Figueiredo is with Faculty of Engineering, Universidade Lusófona de Humanidades e Tecnologias, Campo Grande 376, Lisbon 1749-024, Portugal. $\mathrm{He}$ is also with CONSTRUCT - Institute of R\&D in Structures and Construction, R. Dr. Roberto Frias s/n, Porto 4200-465, Portugal.

N. M. M. Maia is with LAETA, Institute of Mechanichal Engineering, Instituto Superior Técnico, Universidade de Lisboa, Lisbon 1049-001, Portugal

A preliminary version of this paper was presented in XXXVI Simpósio Brasileiro de Telecomunicações e Processamento de Sinais (SBrT'18), Campina Grande, PB, Brazil, September 16-19, 2018 [1].

Digital Object Identifier: 10.14209/jcis.2019.7 can be measured by employing different types of monitoring systems, damage-sensitive features can be then extracted and used to assess early and progressive structural damage via appropriate data treatment.

Frequency response functions (FRFs) play an important role in the vibration-based damage detection area [4]. Many works have been employed the FRFs as features to generate some kind of damage indicators (DIs) that reveal the structural condition of monitored structures [5]-[8]. However, the need for measuring the excitation forces arises as a remarkable limitation on the application of FRFs in real-world SHM solutions.

As an alternative, transmissibility measurements have been widely explored as features in damage detection for SHM [9][12], due to their output-only nature [13]-[15], which implies the need for measuring only the response signals, without any knowledge about the excitation forces.

Considering the successful use of transmissibilities to distinguish between undamaged and damaged conditions of monitored structures, instead of generating a DI directly from the transmissibility measurements, several studies have been attempting to combine FRFs and transmissibilities with machine learning (ML) algorithms to detect structural damage.

The combination between FRFs or transmissibilities and artificial neural networks (ANN) has been widely investigated. In a first manner, the transmissibilities are the input of a complex ANN that should detect damage simulated as stiffness changes in a structure [16], [17]. However, the number of spectral lines of the transmissibilities defines the number of input nodes of the ANN, which may lead to an expensive computational load in terms of algorithm training along with an information criterion to select the neural architecture. In other attempt, the FRFs, computed from a monitored railway wheel, have their dimensionality reduced via principal component analysis (PCA) and this reduced form is the input of the ANN [18]. Although acceptable results were achieved with this technique, a drawback appears when the FRFs or transmissibilities from undamaged and damaged cases must be known in advance by PCA, as damaged conditions might be prohibitive to simulate in some practical cases.

An approach that uses outlier analysis, density estimation and auto-associative neural network combined with measured transmissibilities was proposed to assess damage in aerospace structures [19]-[21]. In this case, some parts of the transmissibility measurements are selected as features in a visual manner, which may not be generalized to other health monitoring cases. 
More recently, FRFs and transmissibilities were linked to the approach based on Mahalanobis squared distance (MSD) to determine the structural condition of a monitored beam via different types of DIs [22]. Similar to a first proposal [23], the MSD algorithm presents problems, namely numerical errors to compute a large covariance matrix, when all spectral lines of the damage-sensitive features are considered. Note that the ML algorithms, such as MSD and ANN, often work with a large number of observations (or measurements) and a small dimensionality (or spectral lines).

PCA and its nonlinear version, kernel PCA (KPCA), have been applied to reduce the dimensionality of the original features such that a trade-off should be reached in the sense that the appropriate dimensionality needs to be not only large enough to account for all normal condition but also small enough to be as sensitive as possible to damage [24]-[26]. To reach this appropriate reduced form of transmissibilities, this paper proposes an output-only structural damage detection method in the context of a statistical pattern recognition (SPR) paradigm. In this paradigm, the proposed method is divided into two phases [27], [28]: feature extraction and feature classification. In the first phase, the dimensionality of the transmissibilities is reduced by application of KPCA. In the second phase, based on outlier detection, DIs are generated for new transmissibilities considering the data model computed in the first phase. The proposed method is validated on a monitored steel beam, under different structural conditions, where the damage classification performance is evaluated based on false-positive indications of damage (Type I errors) and false-negative ones (Type II errors).

The remainder of this study is organized as follows. In section III a brief background related to transmissibility measurements is emphasized and the feature extraction and feature classification phases of the proposed method are described. A description of the test structure is provided in section III. along with a summary of the validation scenarios and their measurement sets. Experimental results on measurement sets from three scenarios are discussed in section IV] a comparison with the PCA algorithm is also presented. Finally, section V highlights a discussion related to the strengths and challenges of the proposed method.

\section{OUTPUT-ONLY METHOD}

This section deals with the background and unsupervised phases related to the method proposed in this paper. First, a brief background related to the transmissibility measurements is introduced. Second, in the feature extraction or training phase, the dimensionality of the transmissibilities is reduced by application of KPCA. In the feature classification or test phase, the damage assessment of the structure is performed by taking into account the generalization of the reduced dimensionality to new transmissibility measurements.

\section{A. Transmissibility measurements}

The motivation of using the transmissibilities to detect structural damage relies on the assumption that they are local quantities [10], which suggests a high sensitivity to detect changes in the structural dynamics caused by some kind of damage. The detection of any progressive damage implies a regular monitoring of the structure at certain time intervals.

Transmissibility measurements are defined as relations between motion responses and motion reference-responses [29]. In practice, it is often convenient to obtain the transmissibilities without the knowledge of the excitation forces. In particular, the direct or scalar transmissibility measurement $T_{i, j}(w)$ between an output $i$ and and reference-output $j$ is defined as the ratio between the two response spectra,

$$
T_{i, j}(w)=\frac{Y_{i}(w)}{Y_{j}(w)},
$$

where $Y_{i}(w)$ and $Y_{j}(w)$ are the complex amplitudes of the responses $y_{i}(t)$ and $y_{j}(t)$, respectively, for a harmonic force applied at a given coordinate, and for each frequency $w$.

In general, for a random input and considering an stationary stochastic process, a transmissibility measurement can be estimated in several manners. The most common option is using an output-only $\mathrm{H}_{1}$ estimator by dividing an estimate of the cross-power spectrum $S_{i, j}(w)$ between the output $Y_{i}(w)$ and the reference-output $Y_{j}(w)$ by an estimate of the autopower spectrum $S_{j, j}(w)$ from the reference-output $Y_{j}(w)$,

$$
T_{i, j}(w)=\frac{S_{i, j}(w)}{S_{j, j}(w)} .
$$

A set of transmissibilities is acquired by measuring responses on all coordinates and directions of interest at the structure divided by the reference response from the same fixed measurement coordinate.

It is worth noting that the phases described in the following are unsupervised in the sense that the training or feature extraction is performed only with undamaged data that should cover nearly all the operational and environmental effects and the test or feature classification is performed with data from any structural condition (undamaged or damaged). Thus, one should consider a training set, $\mathbf{X} \in \mathbb{R}^{n \times d}$, with d-dimensional transmissibilities from $n$ different conditions when the structure is undamaged and a test set, $\mathbf{Z} \in \mathbb{R}^{l \times d}$, where $l$ is the number of transmissibilities from the undamaged and/or damaged conditions.

\section{B. Feature extraction}

For the feature extraction phase, the KPCA algorithm [30] is used to reduce the dimensionality of the transmissibility measurements. Let $\mathcal{X} \in \mathbb{R}^{d}$ be the input space such that the transmissibilities $\mathbf{x}_{i} \in \mathcal{X}, i=1, \ldots, n$. Every transmissibility $\mathbf{x}$ is then mapped to a $d_{\phi}$-dimensional feature space $\mathcal{H}$ by applying the mapping functions $\phi_{m}, m=1, \ldots, d_{\phi}$, where

$$
\phi(\mathbf{x})=\left[\begin{array}{llll}
\phi_{1}(\mathbf{x}) & \phi_{2}(\mathbf{x}) & \ldots & \phi_{d_{\phi}}(\mathbf{x})
\end{array}\right]^{\top} .
$$

By employing the kernel trick [31], $\mathcal{K}: \mathcal{X} \times \mathcal{X} \mapsto \mathbb{R}$ is defined as a positive semi-definite scalar kernel function satisfying for all $\mathbf{x}_{i}, \mathbf{x}_{j} \in \mathcal{X}$,

$$
\mathcal{K}\left(\mathbf{x}_{i}, \mathbf{x}_{j}\right)=\phi\left(\mathbf{x}_{i}\right)^{\top} \phi\left(\mathbf{x}_{j}\right) .
$$


$\mathcal{K}(\cdot)$ defines an inner product that allows to map the transmissibilities implicitly to a high-dimensional kernel space. Let

$$
\mathbf{\Phi}=\left[\begin{array}{llll}
\phi\left(\mathbf{x}_{1}\right) & \phi\left(\mathbf{x}_{2}\right) & \ldots & \phi\left(\mathbf{x}_{n}\right)
\end{array}\right]
$$

be the $d_{\phi} \times n$ matrix of the mapped transmissibilities and $\mathbf{K}=\boldsymbol{\Phi}^{\top} \boldsymbol{\Phi}$ be the $n \times n$ kernel (Gram) matrix. According to Mercer's theorem, any continuous, symmetric, and positive semi-definite function that maps $\left(\mathbf{x}_{i}, \mathbf{x}_{j}\right)$ onto a highdimensional feature space can represent a kernel [32]. The kernel trick then consists of specifying the kernel $\mathcal{K}(\cdot)$ instead of the mapping $\phi$. Herein, a Gaussian kernel [33] is employed,

$$
\mathcal{K}\left(\mathbf{x}_{i}, \mathbf{x}_{j}\right)=\exp \left(-\frac{\left\|\mathbf{x}_{i}-\mathbf{x}_{j}\right\|^{2}}{2 \sigma^{2}}\right),
$$

where this kernel implicitly defines a high-dimensional feature space with a bandwidth $\sigma^{2}$.

To avoid that the first principal component becomes much larger than the other components, the kernel matrix $\mathbf{K}$ should be replaced by a centered version [30],

$$
\mathbf{K} \rightarrow \mathbf{K}-\frac{\mathbf{1}_{\mathbf{n}}}{n} \mathbf{K}-\mathbf{K} \frac{\mathbf{1}_{\mathbf{n}}}{n}+\frac{\mathbf{1}_{\mathbf{n}}}{n} \mathbf{K} \frac{\mathbf{1}_{\mathbf{n}}}{n},
$$

with $\mathbf{1}_{\mathbf{n}}$ as the $n \times n$ matrix composed of ones.

The eigenvalues $\boldsymbol{\Sigma}$ and the corresponding eigenvectors $\mathbf{U}$ can be then derived by using singular value decomposition (SVD) to solve the generalized eigenvalue problem [30],

$$
\mathbf{K U}=\mathbf{U} \boldsymbol{\Sigma} .
$$

Afterwards, the $\boldsymbol{\Sigma}_{1}$ and $\mathbf{U}_{1}$ should be defined as follows,

$$
\boldsymbol{\Sigma}=\left[\begin{array}{ll}
\boldsymbol{\Sigma}_{1} & \boldsymbol{\Sigma}_{2}
\end{array}\right], \boldsymbol{\Sigma}_{1} \in \mathbb{R}^{r \times r} ; \mathbf{U}=\left[\begin{array}{ll}
\mathbf{U}_{1} & \mathbf{U}_{2}
\end{array}\right], \mathbf{U}_{1} \in \mathbb{R}^{n \times r},
$$

where $\boldsymbol{\Sigma}_{1}$ comprises the $r$ largest eigenvalues and $\mathbf{U}_{1}$ the corresponding eigenvectors. The transmissibility measurements $\mathbf{X}$ can be then represented in a reduced form as the transpose of

$$
\mathbf{X}_{p}=\sqrt{\Sigma_{1}} \mathbf{U}_{1}^{\top}
$$

In addition, the estimation of parameters required for the KPCA algorithm is discussed in the following. There are multiple methods to optimize the bandwidth parameter $\sigma^{2}$ of the Gaussian kernel [34]. However, those methods require that $n \geq d$ and when transmissibility measurements are used, often $n<d$. Thereby, a rule-of-thumb is employed,

$$
\mathbf{v}=\operatorname{var}(\mathbf{X}), \sigma^{2}=\frac{n}{d} \sum_{i=1}^{d} v_{i},
$$

where $\operatorname{var}(\mathbf{X})$ is the variance of the training data. Several criteria have been proposed to determine the number of principal components $r$ retained in the high-dimensional feature space [35]. In this study, $r$ is derived to comprise nearly all normal variability of the training data,

$$
\sqrt{\frac{\sum_{i=1}^{r} \boldsymbol{\Sigma}_{i, i}}{\sum_{i=1}^{n} \boldsymbol{\Sigma}_{i, i}}} \geq 0.99,
$$

being $\boldsymbol{\Sigma}_{i, i}$ the $i$-th diagonal element of $\boldsymbol{\Sigma}$. Note that the variance retained in the standard PCA is usually 0.9-0.95 [35]. On the other hand, the KPCA has been often used with 0.99 because there are potentially $n$ nonzero principal components for the Gaussian kernel.

\section{Feature classification}

Since an undamaged data model was established in the previous phase by training the KPCA, in this phase a DI is generated to any new transmissibility measurement $\mathbf{z}_{i} \in \mathbb{R}^{d}$, $i=1, \ldots, l$.

First, a new transmissibility measurement should be mapped onto the high-dimensional kernel space in the form of $\boldsymbol{\Phi}\left(\mathbf{z}_{i}\right)^{\top} \boldsymbol{\Phi}$ (or $\boldsymbol{\Phi}^{\top} \boldsymbol{\Phi}\left(\mathbf{z}_{i}\right)$ ), by using $\mathbf{X}$ and $\mathbf{z}_{i}$ in Equation 6 Besides, a centering should be performed, such as,

$\boldsymbol{\Phi}\left(\mathbf{z}_{i}\right)^{\top} \boldsymbol{\Phi} \rightarrow \boldsymbol{\Phi}\left(\mathbf{z}_{i}\right)^{\top} \boldsymbol{\Phi}-\frac{\check{\mathbf{1}}_{\mathbf{n}}}{n} \mathbf{K}-\boldsymbol{\Phi}\left(\mathbf{z}_{i}\right)^{\top} \boldsymbol{\Phi} \frac{\mathbf{1}_{\mathbf{n}}}{n}+\frac{\check{\mathbf{1}}_{\mathbf{n}}}{n} \mathbf{K} \frac{\mathbf{1}_{\mathbf{n}}}{n}$,

with $\check{\mathbf{1}}_{\mathbf{n}}$ as the $l \times n$ matrix where all elements are equal to 1 .

Second, the eigenvectors $\mathbf{U}_{1}$ should be replaced by a normalized version,

$$
\mathbf{u}_{m} \rightarrow \frac{\mathbf{u}_{m}}{\sqrt{\boldsymbol{\Sigma}_{m, m}}}, m=1, \ldots, r,
$$

being the transmissibilities used in the test phase, $\mathbf{Z}$, represented in a reduced form as

$$
\mathbf{Z}_{p}=\boldsymbol{\Phi}\left(\mathbf{z}_{i}\right)^{\top} \boldsymbol{\Phi} \mathbf{U}_{1} .
$$

A DI is then generated for the $i$-th new transmissibility measurement as follows,

$$
\mathrm{DI}\left(\mathbf{z}_{i}\right)=\boldsymbol{\Phi}\left(\mathbf{z}_{i}\right)^{\top} \boldsymbol{\Phi} \mathbf{U}_{1} \mathbf{U}_{1}^{\top} \boldsymbol{\Phi}^{\top} \boldsymbol{\Phi}\left(\mathbf{z}_{i}\right) .
$$

Finally, any DI can be classified by a threshold estimated from the training data, considering a given level of significance. The general idea is an anomaly or outlier detection strategy. By considering the undamaged model estimated in the training phase, when a new observation comes from the undamaged conditions learned by the model, it should be classified as a normal behavior, whereas if a new observation comes from any unknown condition, it should be classified as a possible anomaly or abnormal behavior.

\section{The possibility of clustering in high-dimensional space}

It is worth to mention the possibility of clustering in the high-dimensional space learned by KPCA. The theory provided for feature extraction in this study can be extended to classify undamaged and damaged cases via a density-based procedure which is widely known in computer vision as mean 
shift clustering (MSC) [36]. MSC is a nonparametric algorithm that does not require prior knowledge of the number of clusters and does not constrain their shapes. It iteratively discovers the modes in the data by maximizing the kernel density estimate. In addition, the number of clusters is automatically estimated by the number of discovered modes.

Although the MSC is applied in the Euclidean or original space, it can be formulated to act in high-dimensional spaces with aid of a kernel function [37]. The motivation to the aforesaid adaptation is that in the original space if the clusters are not linearly separable and/or the distance metric is not adequate, poor clustering performance is usually obtained. On the other hand, in high-dimensional spaces as the one mapped by KPCA, data becomes linearly separable and meaningful cluster can be achieved.

In the context of damage detection, a kernel MSC comprises the following steps: i) feature extraction via KPCA on training data; ii) application of MSC in the learned space to estimated the undamaged modes or clusters; iii) mapping of test data onto the learned space and afterwards application of MSC on them to classify as undamaged when they are close to the undamaged modes and damaged when they are far from these clusters. In the last step, with the application of MSC on mapped test data, the normal cases should converge to the undamaged modes and abnormal ones should diverge from them forming new modes that denote new structural conditions. These new conditions might be classified as damage.

\section{TEST STRUCTURE AND DATA SETS}

For the experimental validation of the proposed method, a steel beam was used, with rectangular cross-section, dimensions $L \times b \times h=1002 \times 35 \times 6 \mathrm{~mm}^{3}$ and weight 1.740 $\mathrm{kg}$. Two inextensible cables simulating "free-free" support conditions suspended the test structure. The test structure was excited at locations 3 and 12 with a pseudo-random signal by a Brüel \& Kjaer 4809 shaker, powered by a Brüel \& Kjaer 2706 power amplifier. The force was transmitted through a stinger and measured by a Brüel \& Kjaer 8200 force transducer; the responses were measured by 23 piezoelectric CCLD accelerometers (equally spaced coordinates). The response signals were fed into the multi-channel data acquisition unit Brüel \& Kjaer 2816 (PULSE) and analyzed with the Labshop 6.1 Pulse software. The experimental setup is shown in Figure 1 and more details can be found in Sampaio et al [8].

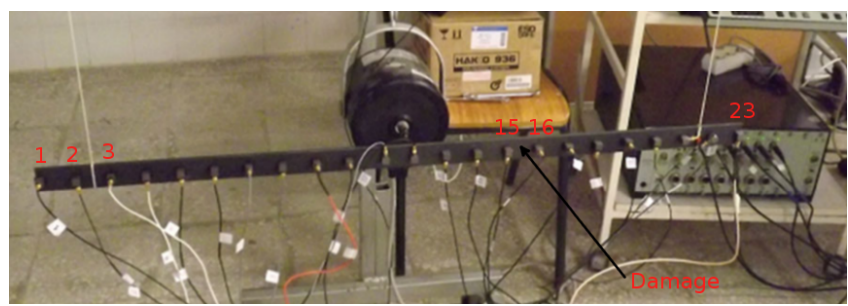

Fig. 1. Experimental setup with the identification of the accelerometers and damage location.

The damage was simulated with a reduction in the height $h$ by a saw cut, as depicted in Figure 2 Basically, saw cuts, with several depths, were inflicted to the beam between locations 15 and 16 to create nine damage levels, as synthesized in Table (1) and exemplified in Figure 3 for the second level of damage.

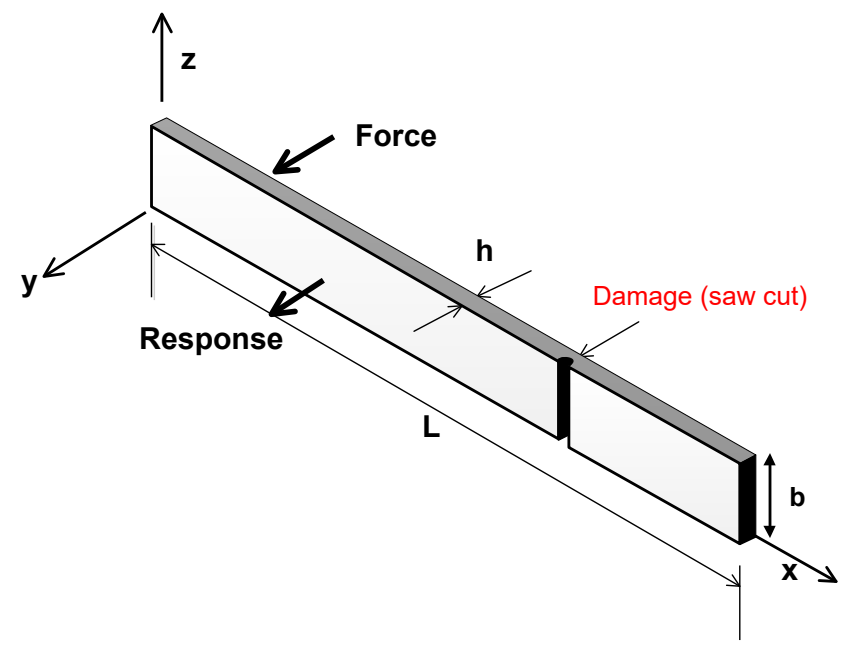

Fig. 2. Beam in transverse vibration.

TABLE I

DAMAGE LEVELS INFLICTED TO THE EXPERIMENTAL BEAM BY SAW CUTS

\begin{tabular}{lll}
\hline Damage level & Width $(\mathrm{mm})$ & Depth $(\mathrm{mm})$ \\
\hline D01 & 1.0 & 0.5 \\
D02 & 1.0 & 1.0 \\
D03 & 1.0 & 1.25 \\
D04 & 1.0 & 1.6 \\
D05 & 1.0 & 3 \\
D06 & 1.0 & 3.5 \\
D07 & 1.0 & 4 \\
D08 & 1.0 & 4.5 \\
D09 & 1.0 & 5 \\
\hline
\end{tabular}

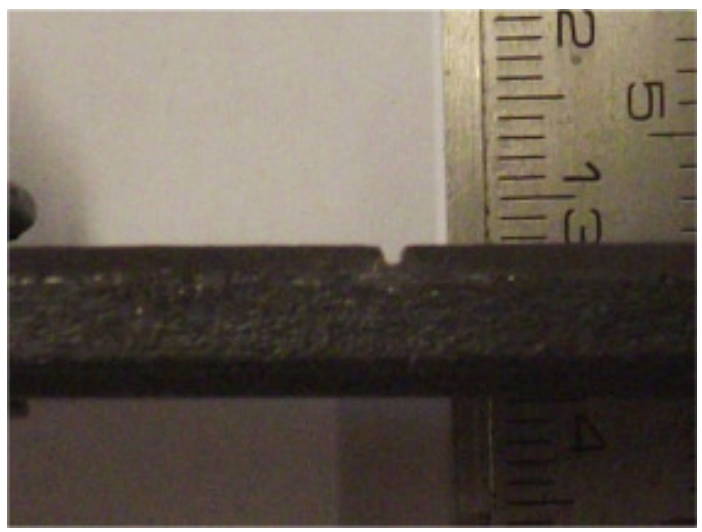

Fig. 3. Detail of the second level of damage caused by a saw cut [8].

Therefore, from Table I one can infer that the response signals from the accelerometers deployed on the beam were measured in ten conditions; the undamaged or baseline one (D00), and the nine levels of damage (D01 to D09) inflicted in the middle of the locations 15 and 16. Furthermore, the frequency range used for the analysis of the beam was $0-800$ 
$\mathrm{Hz}$ (3200 spectral lines) and 15 averages have been taken to acquire the accelerations.

Firstly, according to the location of the excitation force, two scenarios are considered; a force at location 3 and a force at location 12. For both cases, 30 measurement sets have been performed from the instrumented beam. The first three measurement sets are related to the undamaged beam (Baseline condition - BC). The following 27 measurement sets correspond to the nine saw cuts of the beam with three measurements sets each (Damaged condition - DC).

In the aforementioned case, considering all possible combinations to output and reference-output, 506 transmissibilities can be generated for each measurement set. For convenience, only 100 transmissibilities are selected at random from each measurement set, yielding 3000 transmissibilities to compose the training and test measurement sets, where the first 300 are transmissibilities from the undamaged condition and the last 2700 transmissibilities corresponding to the nine levels of damage. The training data is composed of $90 \%$ of the transmissibility measurements from the undamaged condition. The remaining $10 \%$ of the transmissibility measurements are used during the test phase to make sure that the DIs do not fire off before the damage starts and to evaluate the level of generalization of the proposed method. The test data is composed of all the measurement sets, even the ones used during the training phase. Note that the training process is unsupervised, which imposes serious limitations for a crossvalidation procedure.

A third scenario was carried out with the excitation force applied at location 12, where 21 measurement sets have been performed from the monitored beam. Once again, the first three measurement sets comprise the undamaged condition (M00). The following three measurement sets are different measurements under the following condition: an added bolt with $2 \mathrm{~g}$ between locations 17 and 18 (M01). The next three measurement sets are different measurements under the following condition: an added nut, with $0.7 \mathrm{~g}$, to the previous condition of the beam (M02). And so on until 5 nuts are added (M03 to M06). An example of the third scenario is presented in Figure 4 The training and test data are composed in the same manner as described to the first two scenarios.

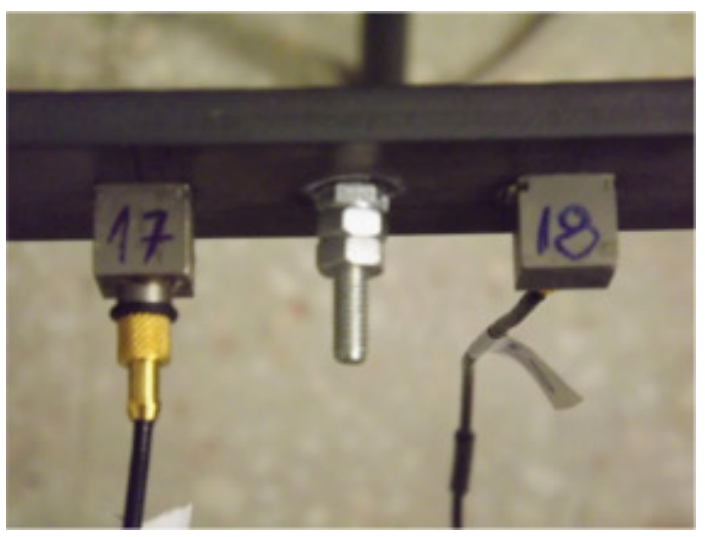

Fig. 4. Example of the third scenario (an added mass) [8].

\section{RESULTS AND DISCUSSION}

In this section, the proposed method is demonstrated by application to the aforementioned scenarios and their measurement sets, which were obtained by monitoring a steel beam under different structural conditions. The methods based on the KPCA and PCA algorithms are employed and their damage detection performances are compared. Besides, the application of kernel MSC is briefly demonstrated on the first scenario.

\section{A. First scenario: force at location 3 and nine levels of damage}

Examples of transmissibilities and FRFs derived from the monitored beam under different conditions are shown in Figure 5 Although, in contrast to FRFs, the peaks in the magnitude of the transmissibility measurements do not at all coincide with the resonances of the structural system, there are notable differences between the undamaged condition and the maximum level of damage for both transmissibilities and FRFs. However, the large number of spectral lines and small number of measurements make the transmissibilities inappropriate to be suitably processed by the ML algorithms. Thereby, this fact highlights the need for dimensionality reduction.
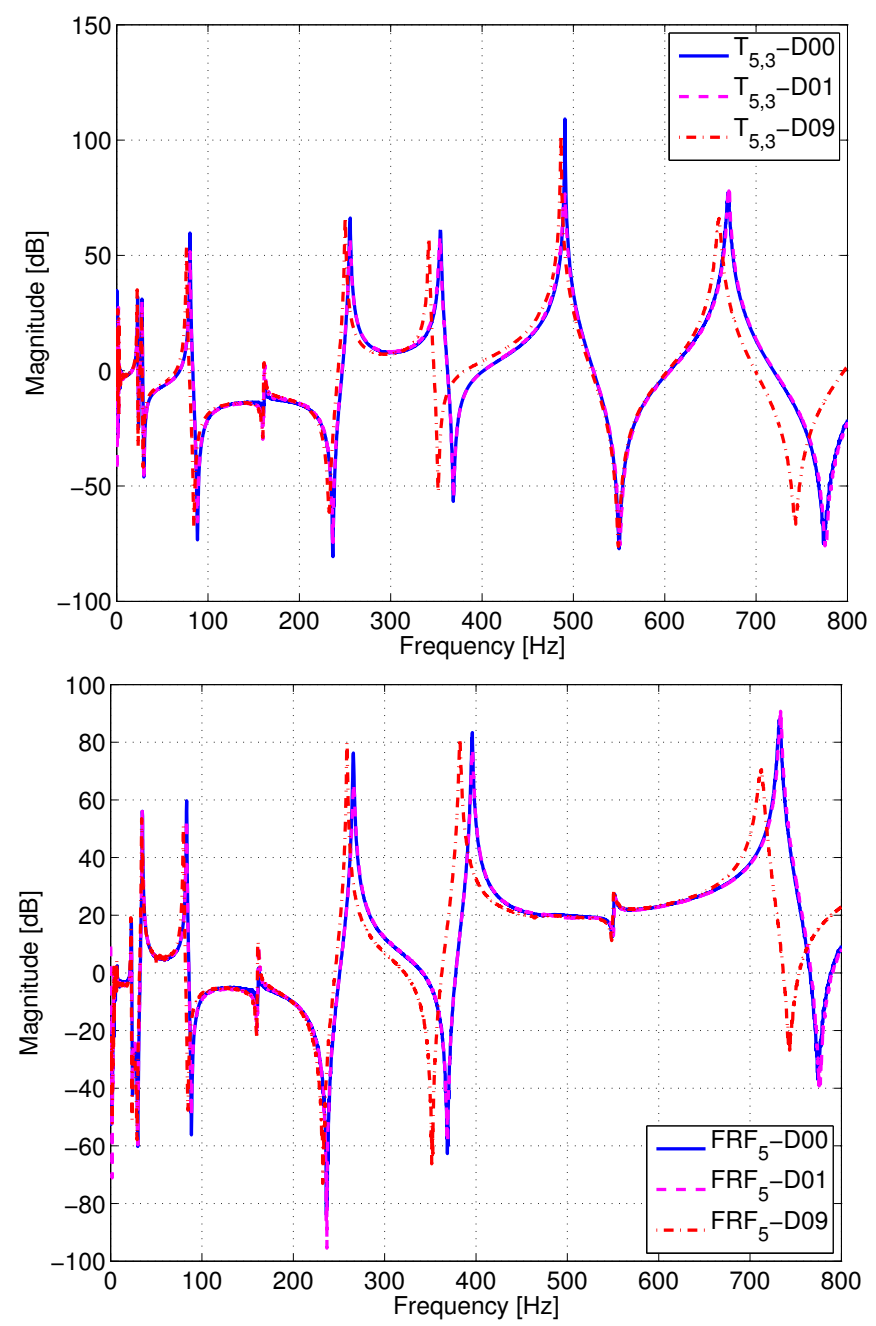

Fig. 5. First scenario: $T_{5,3}(w)$ (top) and $\operatorname{FRF}_{5}(w)$ (bottom) for three different conditions. 
After the algorithms have reduced the dimensionality of the transmissibility measurements, the DIs derived from the KPCA and PCA algorithms are shown in Figure 6, along with a threshold defined for a level of significance of $5 \%$ over the training data. The KPCA, which selected 181 principal components, can maintain a monotonic relationship between the progressive level of damage and the amplitude of the DI and minimizes quite well the Type I and II errors. In opposition, the PCA, which selected only 14 principal components, fails to achieve the monotonic relationship and exhibits many false-negative indications of damage. The performances of the algorithms are summarized in Table III. where approximately 13 Type I errors are expected due to the threshold selected for $95 \%$ of confidence in the training data and the disparity between the total errors from both algorithms is evident.
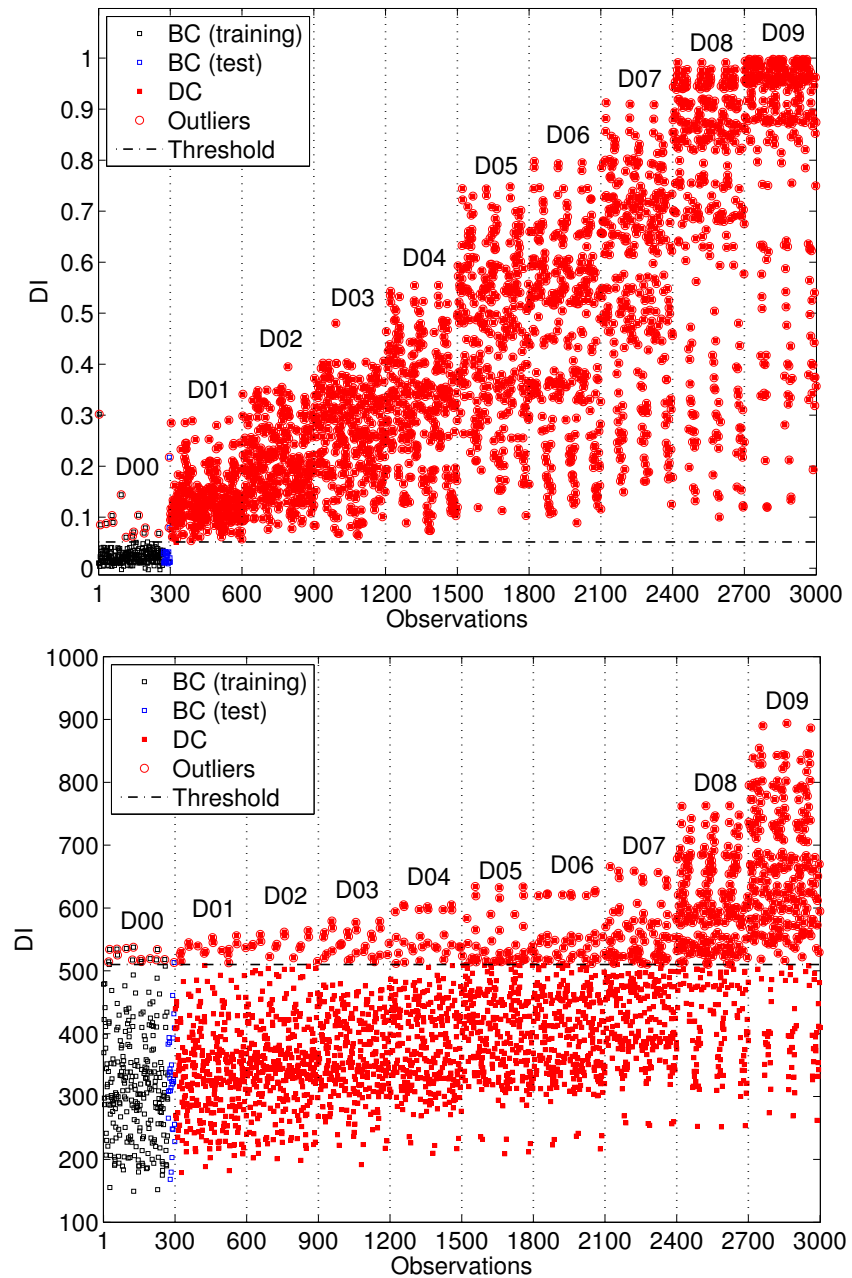

Fig. 6. First scenario: outlier detection based on the KPCA (top) and PCA (bottom) algorithms.

TABLE II

FIRST SCENARIO: NUMBER AND PERCENTAGE OF TYPE I AND TYPE II ERRORS

\begin{tabular}{llll}
\hline \multirow{2}{*}{ Algorithm } & Error & & \\
\cline { 2 - 4 } & Type I & Type II & Total \\
\hline KPCA & $15(5.00 \%)$ & $1(0.037 \%)$ & $16(0.53 \%)$ \\
PCA & $14(4.67 \%)$ & $2032(75.26 \%)$ & $2046(68.20 \%)$ \\
\hline
\end{tabular}

The large difference between the performances of the algorithms is explained through Figure 7 In the high-dimensional feature space mapped by KPCA, the principal components are distributed in a more representative manner than those distributed onto the feature space projected by PCA. Thus, the transmissibilities that have been reduced by KPCA, in the training phase, from 3200 to 181 dimensions can be generalized to new transmissibilities, ensuring an adequate dimension to detect structural anomalies, whereas the reduction from 3200 to 14 dimensions by PCA impacted in an overfitting regarding the training measurement sets.
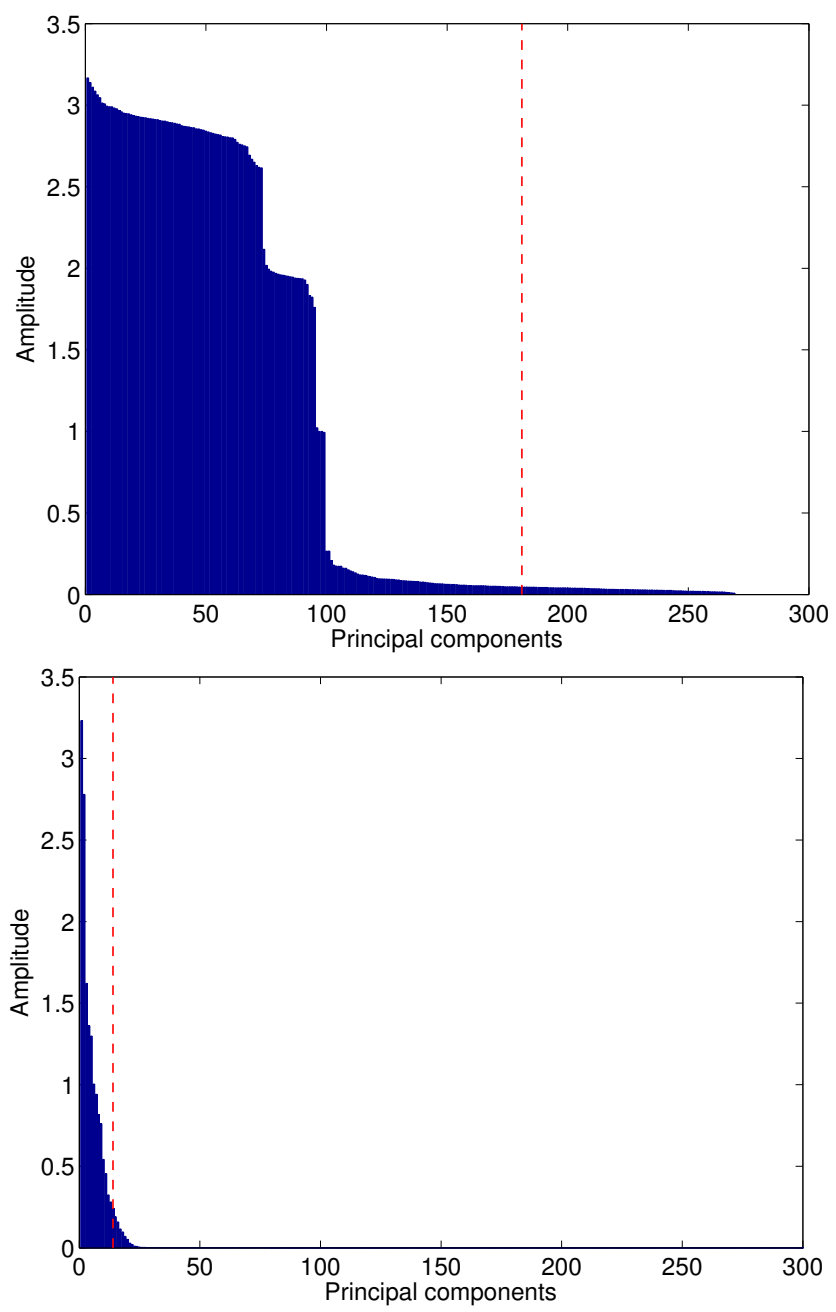

Fig. 7. First scenario: distribution of principal components for the KPCA (top) and PCA (bottom) algorithms. The number of principal components selected is highlighted by a red dashed line.

Often the input or Euclidean space of transmissibility measurements imposes limitations for a suitable processing in terms of damage classification. For instance, in this space is hard to discover clusters or density modes that group together transmissibilities from the same structural conditions, due to the many spectral lines of these measurements mainly. Thereby, the learned space via KPCA can be an alternative for the aforesaid task. Figure 8 shows non-separable clusters in the input space and modes estimated by kernel MSC in the feature space mapped with KPCA. Unlike the Euclidean 
space, the modes in the mapped space are linearly separable and readily computed. Note that, for visualization purposes, only two dimensions and ten test transmissibilities for each condition (D00-D09) were considered.
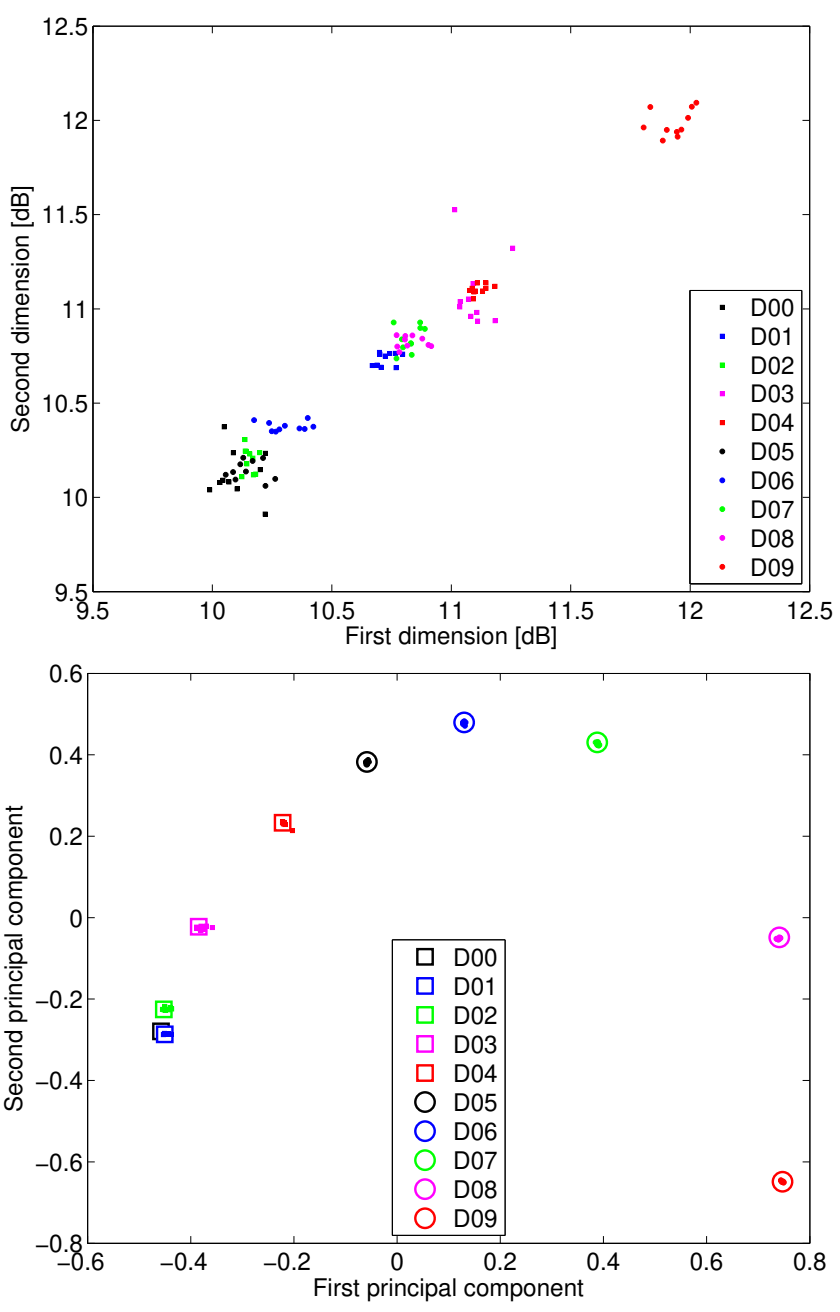

Fig. 8. First scenario: features in Euclidean space (top) and learned kernel space after the application of kernel MSC (bottom). Transmissibilities and modes are markers with colored and non-colored face, respectively.

Figure 9 provides a better understanding of the clustering procedure. Ten modes were estimated; a baseline one in the training phase and the nine damaged ones in the test phase. Each transmissibility was correct classified according to the condition to which it belongs. It is clear that, after the application of kernel MSC, the transmissibilities converged to the neighborhood of the closest mode. Some transmissibilities from the condition D00 seem belong to D01, but this minor ambiguity is related to the visualization from only two principal component and disappears when one takes into account the contributions of all principal components selected by KPCA. In this mapped kernel space one can also infer the progressive level of damage by comparing the distance between the maximum levels of damage (D08 and D09) with a intermediary one (D07), for example.
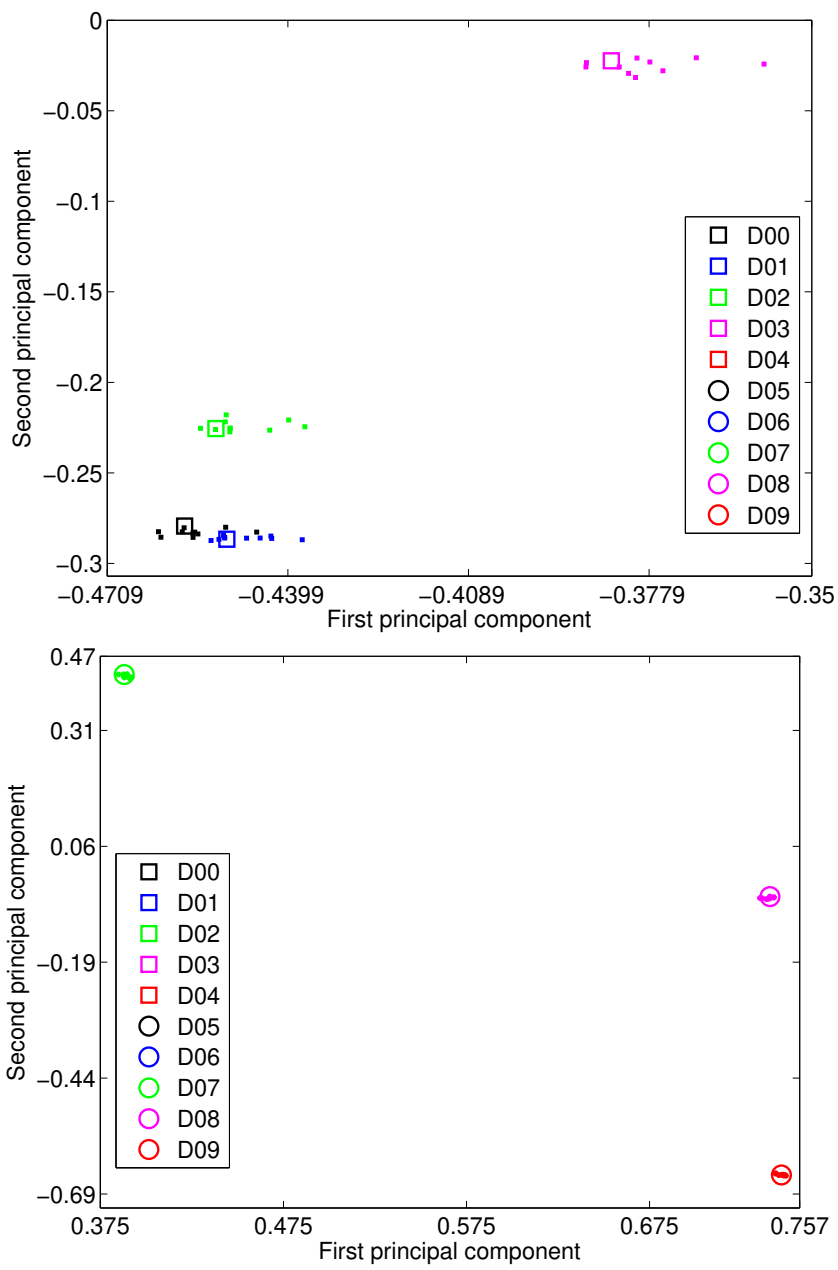

Fig. 9. First scenario: zoom of learned kernel space after the application of kernel MSC. Transmissibilities and modes are markers with colored and non-colored face, respectively.

\section{B. Second scenario: force at location 12 and nine levels of damage}

In the second scenario, examples of FRFs from the monitored beam present relative large differences between the undamaged condition and the maximum level of damage when compared to examples of transmissibilities, as indicated in Figure 10. In this case, despite the relative minor sensitivity of the transmissibilities to damage against FRFs, the former remains useful to distinguish between undamaged and damaged conditions, and its output-only nature plays an important role in SHM solutions where the forces can not be measured.

The DIs computed by the KPCA and PCA algorithms are shown in Figure 11, along with a threshold defined for a level of significance of 5\% over the training data. The KPCA, which selected 133 principal components, once again, can maintain a monotonic relationship between the progressive level of damage and the amplitude of the DI and minimizes quite well the Type I errors. On the other hand, some Type II errors arise mainly in the first level of damage (D01). These additional errors are related to a minor loss of information due to the assumption that the principal components are retained based on $99 \%$ of variance, which implies on the selection of less 

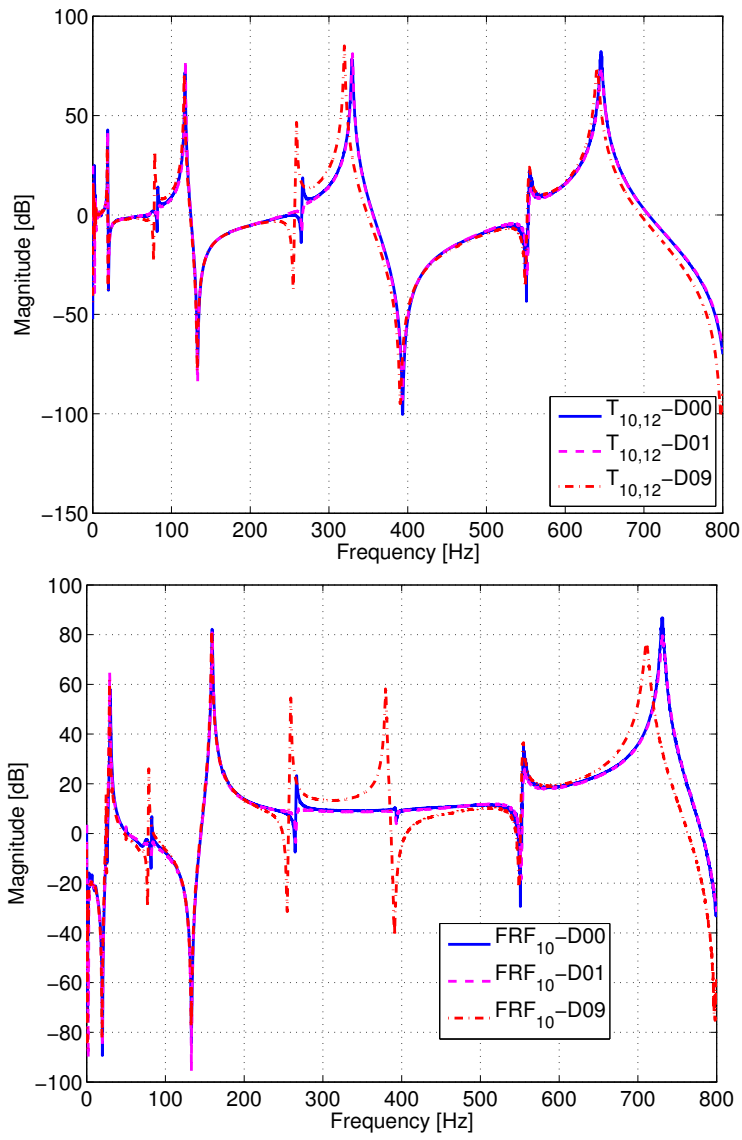

Fig. 10. Second scenario: $T_{10,12}(w)$ (top) and $\operatorname{FRF}_{10}(w)$ (bottom) for three different conditions.

principal components compared to those selected in the first scenario.

By selecting only 10 principal components, the PCA, once again, fails to establish the monotonic relationship and outputs more Type II errors than those presented in the previous scenario. The performances of the algorithms are synthesized in Table [III where in an overall analysis the KPCA has better damage detection performance than the PCA.

For both algorithms, the force at location 12 seems make the minimization of false-negative indications of damage more difficult. A possible reason for this challenge is that the force location becomes more close to the source of damage and few transmissibility measurements selected at random from the nine levels of damage might be generated by the relation between output and reference-output sensors relatively far from the force and source of damage locations. This fact corroborates the increase in the number of Type II errors for this scenario, besides the minor loss of information inherent in the working principles of KPCA.

\section{Third scenario: force at location 12 and added masses}

In contrast to the previous scenarios, this scenario presents examples of transmissibilities and FRFs from the monitored beam which demonstrate minor differences between the undamaged condition and the maximum level of added mass, as evidenced in Figure 12 Therefore, this case is more
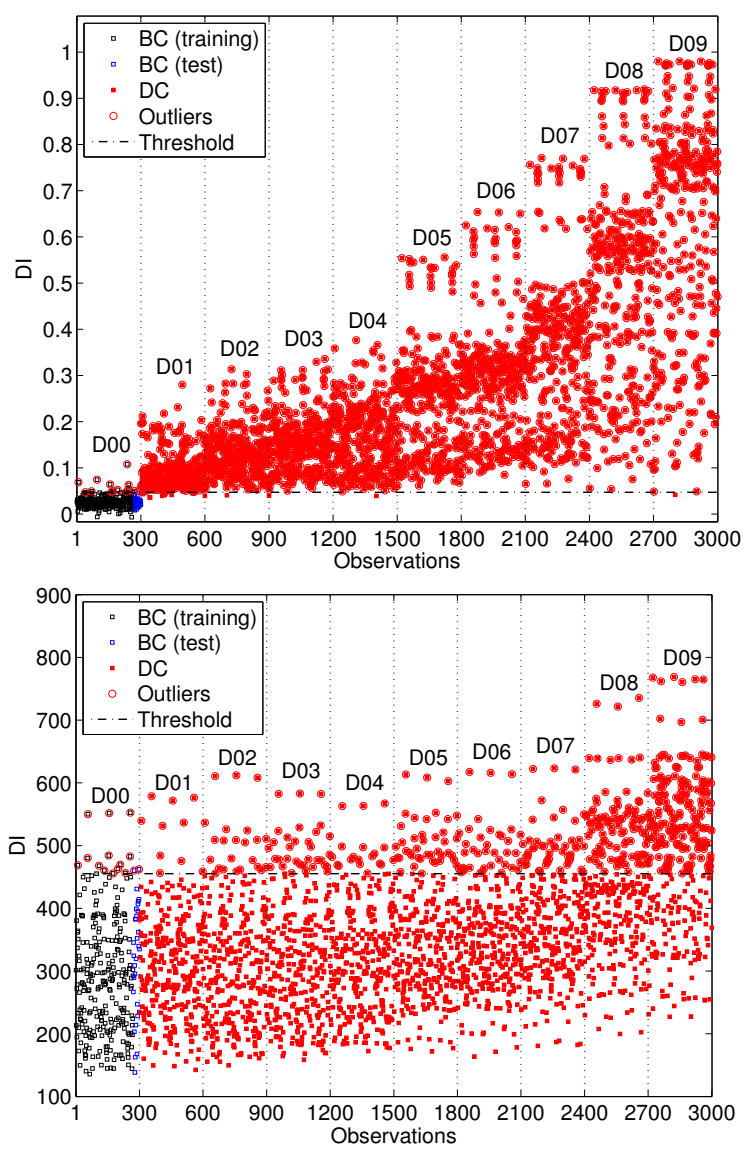

Fig. 11. Second scenario: outlier detection based on the KPCA (top) and PCA (bottom) algorithms.

TABLE III

SECOND SCENARIO: NUMBER AND PERCENTAGE OF TYPE I AND TYPE II ERRORS FOR EACH ALGORITHM.

\begin{tabular}{llll}
\hline \multirow{2}{*}{ Algorithm } & Error & & \\
\cline { 2 - 4 } & Type I & Type II & Total \\
\hline KPCA & $14(4.67 \%)$ & $15(0.56 \%)$ & $29(0.97 \%)$ \\
PCA & $15(5.00 \%)$ & $2207(81.74 \%)$ & $2222(74.07 \%)$ \\
\hline
\end{tabular}

challenging to the unsupervised ML algorithms because the success of these methods depends highly on the sensitivity of the transmissibility measurements (or FRFs). Note that in the SHM literature, the addition of masses is an operational condition which is often assumed to be an undamaged or normal case. In this study, this operational behavior is assumed to be a damaged or abnormal condition only to emphasize the ability of the algorithms to distinguish between the BC (M00) and other conditions (M01-M06).

The DIs computed by the KPCA and PCA algorithms are represented in Figure 13, along with a threshold defined for a level of significance of $5 \%$ over the training data. The KPCA, which selected 133 principal components as expected, satisfactorily minimizes the Type I errors and reaches a monotonic relationship between the progressive level of added mass and the amplitude of the DI. This is an important fact, since the increment of mass, between each level, is very low compared to the total mass of the beam. On the other hand, 

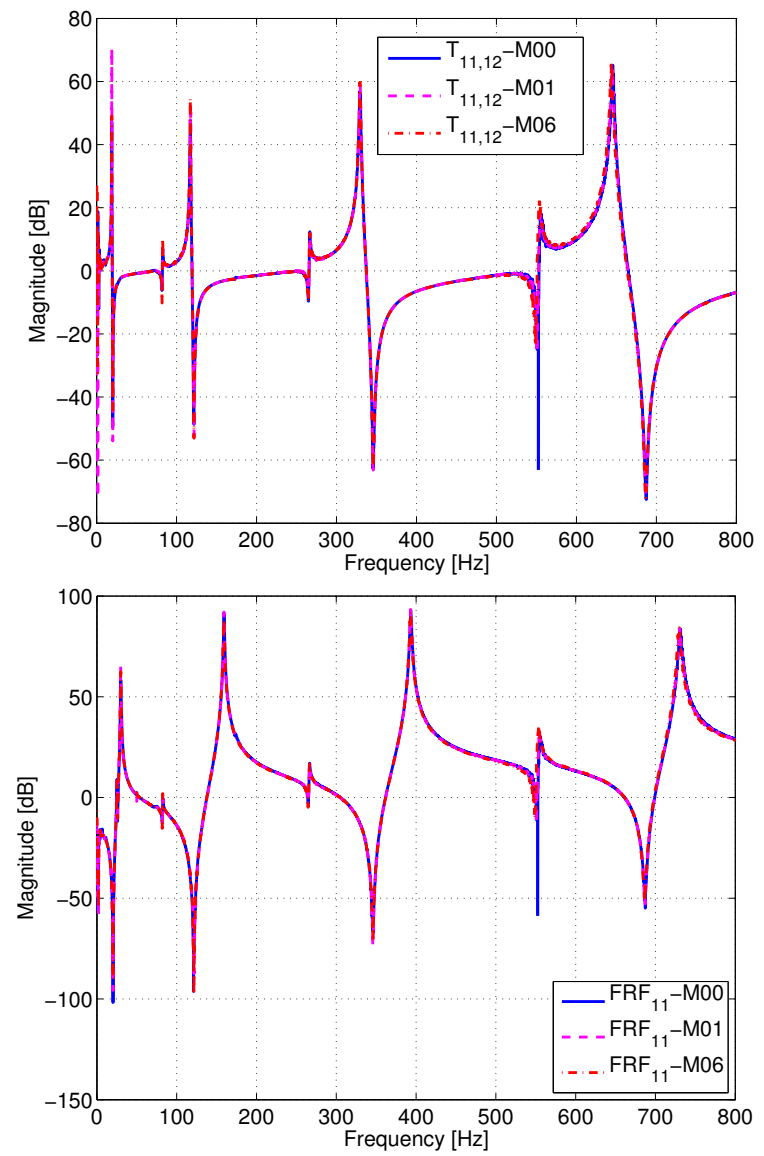

Fig. 12. Third scenario: $T_{11,12}(w)$ (left) and $\operatorname{FRF}_{11}(w)$ (right) for three different conditions.

some Type II errors appear in different levels of added mass and they are mainly related to the differences which are barely visible from the transmissibility measurements under distinct structural conditions.

The worst performance of the PCA is achieved in this scenario where only 10 principal components (as expected) were chosen, which negatively influences the discrimination of two different conditions with imperceptible differences. The algorithm approximately attributes the same amplitude of the DI to different levels of added mass and $92 \%$ of damage cases are misclassified, as shown in Table IV.

The number of principal components selected by KPCA (or PCA) was expected since the force location is the same chosen for the second scenario, i.e., the condition D00 from the second scenario is similar to the condition M00. As the algorithms are unsupervised, their training phases are conducted only with undamaged data and due to D00 and M00 are similar, the numbers of Type I errors in Tables III e IV are identical.

\section{Execution time on measurement sets}

The average execution time for each algorithm on measurement sets from the three scenarios considered in this study is presented in Table V. All the experiments ran on an Intel Core i7-4500U processor $1.8-\mathrm{GHz}(2.4-\mathrm{GHz})$ system with 4 GB of main memory. In an overall analysis, the KPCA
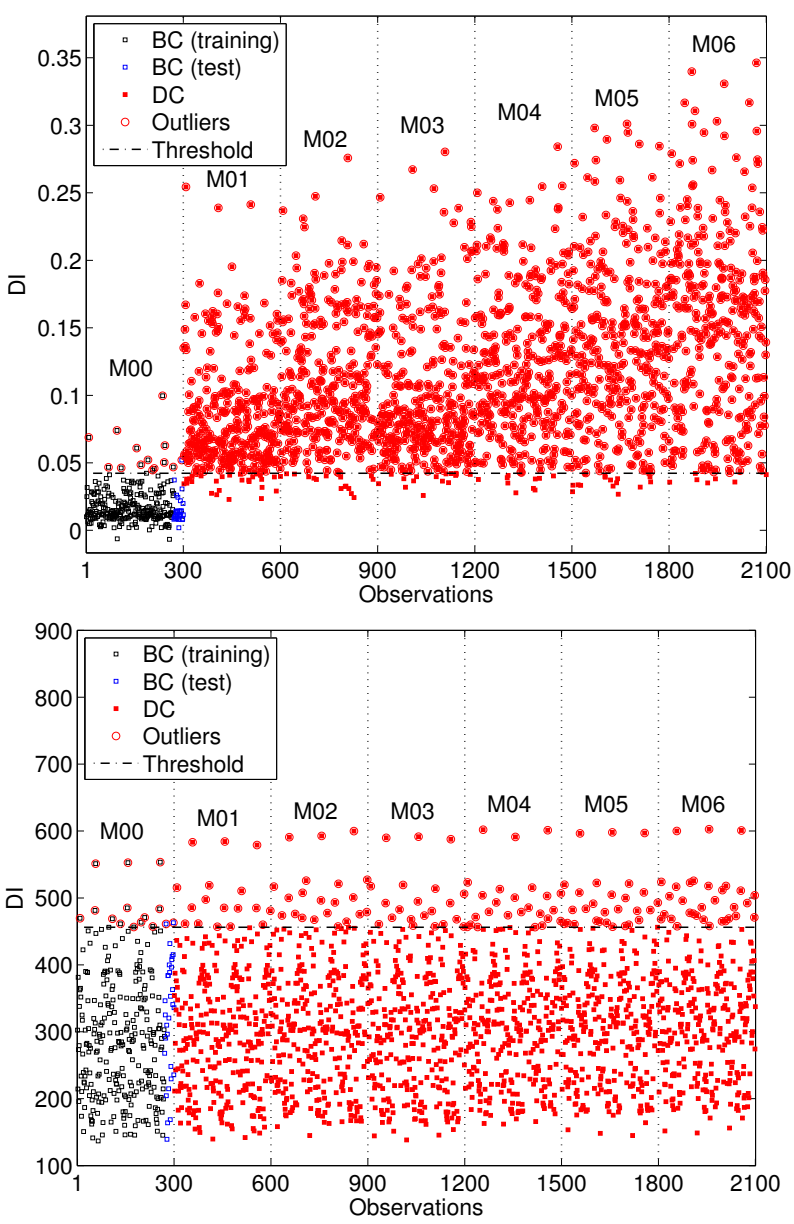

Fig. 13. Third scenario: outlier detection based on the KPCA (top) and PCA (bottom) algorithms.

algorithm has the best time performance for all measurement sets mainly because the kernel matrix has the dimensions equal to the number of transmissibility measurements, which is small compared to the number of spectral lines. Thus, the steps of KPCA regarding the computation of eigenvalues and eigenvectors, as well as the feature classification phase, run very fast.

TABLE IV

THIRD SCENARIO: NUMBER AND PERCENTAGE OF TYPE I AND TYPE II ERRORS FOR EACH ALGORITHM.

\begin{tabular}{llll}
\hline \multirow{2}{*}{ Algorithm } & Error & & \\
\cline { 2 - 4 } & Type I & Type II & Total \\
\hline KPCA & $14(4.67 \%)$ & $92(5.11 \%)$ & $106(5.05 \%)$ \\
PCA & $15(5.00 \%)$ & $1656(92.00 \%)$ & $1671(79.57 \%)$ \\
\hline
\end{tabular}

TABLE V

EXECUTION TIME FOR EACH ALGORITHM ON EACH TEST SCENARIO CONSIDERING TRAINING AND TEST PHASES (AVERAGE FOR 20 EXECUTIONS).

\begin{tabular}{llll}
\hline \multirow{2}{*}{ Algorithm } & \multicolumn{3}{l}{ Average time (seconds) } \\
\cline { 2 - 4 } & First & Second & Third \\
\hline KPCA & 0.281 & 0.283 & 0.199 \\
PCA & 24.805 & 25.063 & 22.317 \\
\hline
\end{tabular}


As mentioned before for the MSD algorithm, the PCA also calculates a large covariance matrix with dimensions equal to the number of spectral lines, which may introduce numerical errors and increase the total execution time of this algorithm.

\section{CONCLUSIONS}

This paper proposed an output-only structural damage detection method, for which transmissibility measurements and ML algorithms were used to assess the condition of monitored structures. Feature extraction and feature classification phases were developed, based on the SPR paradigm, to reduce the dimensionality of transmissibilities via KPCA (training) and to generate a DI that establishes the level of damage for each new transmissibility measurement (test), respectively. By considering three distinct scenarios, the damage classification performance of the proposed method on challenging transmissibility measurement sets from a monitored beam was evaluated and compared to a state-of-the-art method. The extension of the training phase to provide damage classification by clustering was also demonstrated on the first scenario.

The damage detection performances on three different scenarios confirmed that the proposed method is better than the alternative one. When the KPCA is compared to the PCA, the improvement of the mapped feature space proved to have a direct and positive impact on the dimensionality reduction step and consequently damage detection. This explains, in part, the relatively poor performance of the PCA on measurement sets from all scenarios. In addition, the KPCA had better execution time than the PCA on all scenarios due to the appropriate formulation of the former algorithm to work on transmissibility sets with large number of spectral lines and small number of measurements.

Unlike the other approaches from the literature, the outputonly strategy introduced in this study presents two main advantages. First, the transmissibility measurements are processed by KPCA such that an appropriate dimensionality is achieved for ML applications, solving the problem of many spectral lines and small number of measurements. Second, the training and test phases can be seen as separated steps, i.e., in the feature extraction phase, only some part from the undamaged cases should be used to train the KPCA algorithm and in the feature classification phase, any new case (undamaged or damaged) is tested. In other words, after the training phase, the KPCA does not need to know in advance whether a new transmissibility is from the undamaged condition or not.

In general, the choice of parameters, as well as the tuning of them, for ML algorithms becomes very challenging when different scenarios are considered. To deal with this issue, few recommendations were suggested for the KPCA algorithm, taking into account the heterogeneity of monitoring data and parameter settings widely tested and established in the specialized literature. Moreover, the KPCA is sensitive to some loss of information in the high-dimensional feature space as only a certain fraction (herein 99\%) of the normal variability is retained.

As future works, the present method will be tested on other real-world engineering structures and a new method for damage detection will be developed based on kernel MSC.

\section{REFERENCES}

[1] A. Santos, M. F. M. Silva, R. Santos, E. Figueiredo, N. Maia, and J. Costa, "Applicability of an output-only structural damage detection based on transmissibilitymeasurements and kernel principal component analysis," in Proceedings of the XXXVI Simpósio Brasileiro de Telecomunicações e Processamento de Sinais. Campina Grande, Brazil: SBrT, Sep 2018, pp. 851-855. [Online]. Available: https://www. dropbox.com/s/89k2t7swo064afk/Anais_SBrT_2018_v2.pdf?dl=0

[2] C. R. Farrar, S. W. Doebling, and D. A. Nix, "Vibration-based structural damage identification," Philosophical Transactions of the Royal Society of London A: Mathematical, Physical and Engineering Sciences, vol. 359, no. 1778, pp. 131-149, 2001. [Online]. Available: https://doi.org/10.1098/rsta.2000.0717

[3] W. Fan and P. Qiao, "Vibration-based damage identification methods: a review and comparative study," Structural Health Monitoring, vol. 10, no. 1, pp. 83-111, 2011. [Online]. Available: https: //doi.org/10.1177/1475921710365419

[4] N. Maia, J. Silva, E. Almas, and R. Sampaio, "Damage detection in structures: from mode shape to frequency response function methods," Mechanical Systems and Signal Processing, vol. 17, no. 3, pp. 489-498, 2003. [Online]. Available: https://doi.org/10.1006/mssp.2002.1506

[5] R. Sampaio and N. Maia, "Strategies for an efficient indicator of structural damage," Mechanical Systems and Signal Processing, vol. 23, no. 6, pp. 1855-1869, 2009, special Issue: Inverse Problems. [Online]. Available: https://doi.org/10.1016/j.ymssp.2008.07.015

[6] J. Lataire, E. Louarroudi, and R. Pintelon, "Detecting a timevarying behavior in frequency response function measurements," IEEE Transactions on Instrumentation and Measurement, vol. 61, no. 8, pp. 2132-2143, 2012. [Online]. Available: https://doi.org/10.1109/TIM. 2012.2197072

[7] B. Blachowski, A. Swiercz, P. Gutkiewicz, J. Szelek, and W. Gutkowski, "Structural damage detectability using modal and ultrasonic approaches," Measurement, vol. 85, pp. 210-221, 2016. [Online]. Available: https://doi.org/10.1016/j.measurement.2016.02.033

[8] R. Sampaio, N. Maia, R. Almeida, and A. Urgueira, "A simple damage detection indicator using operational deflection shapes," Mechanical Systems and Signal Processing, vol. 72-73, pp. 629-641, 2016. [Online]. Available: https://doi.org/10.1016/j.ymssp.2015.10.023

[9] C. Devriendt, F. Presezniak, G. D. Sitter, K. Vanbrabant, T. D. Troyer, S. Vanlanduit, and P. Guillaume, "Structural Health Monitoring in Changing Operational Conditions Using Tranmissibility Measurements," Shock and Vibration, vol. 17, no. 4-5, pp. 651-675, 2010. [Online]. Available: https://doi.org/10.3233/SAV-2010-0556

[10] N. M. Maia, R. A. Almeida, A. P. Urgueira, and R. P. Sampaio, "Damage detection and quantification using transmissibility," Mechanical Systems and Signal Processing, vol. 25, no. 7, pp. 2475-2483, 2011. [Online]. Available: https://doi.org/10.1016/j.ymssp.2011.04.002

[11] J. García-Barruetabeña and F. Cortés, "Experimental analysis of the vibrational response of an adhesively bonded beam," Measurement, vol. 55, pp. 238-245, 2014. [Online]. Available: https://doi.org/10.1016/ j.measurement.2014.05.011

[12] Y.-L. Zhou, E. Figueiredo, N. Maia, and R. Perera, "Damage Detection and Quantification Using Transmissibility Coherence Analysis," Shock and Vibration, vol. 2015, pp. 1-16, 2015. [Online]. Available: https://doi.org/10.1155/2015/290714

[13] C. Devriendt and P. Guillaume, "The use of transmissibility measurements in output-only modal analysis," Mechanical Systems and Signal Processing, vol. 21, no. 7, pp. 2689-2696, 2007. [Online]. Available: https://doi.org/10.1016/j.ymssp.2007.02.008

[14] —, "Identification of modal parameters from transmissibility measurements," Journal of Sound and Vibration, vol. 314, no. 1-2, pp. 343-356, 2008. [Online]. Available: https://doi.org/10.1016/j.jsv.2007. 12.022

[15] C. Devriendt, G. D. Sitter, and P. Guillaume, "An operational modal analysis approach based on parametrically identified multivariable transmissibilities," Mechanical Systems and Signal Processing, vol. 24, no. 5, pp. 1250-1259, 2010, special Issue: Operational Modal Analysis. [Online]. Available: https://doi.org/10.1016/j.ymssp.2009.02.015

[16] K. Worden, "Structural fault detection using a novelty measure," Journal of Sound and Vibration, vol. 201, no. 1, pp. 85-101, 1997. [Online]. Available: https://doi.org/10.1006/jsvi.1996.0747

[17] Q. Chen, Y. Chan, and K. Worden, "Structural fault diagnosis and isolation using neural networks based on response-only data," Computers \& Structures, vol. 81, no. 22-23, pp. 2165-2172, 2003. [Online]. Available: https://doi.org/10.1016/S0045-7949(03)00295-5 
[18] C. Zang and M. Imregun, "Structural damage detection using artificial neural networks and measured FRF data reduced via principal component projection," Journal of Sound and Vibration, vol. 242, no. 5, pp. 813-827, 2001. [Online]. Available: https: //doi.org/10.1006/jsvi.2000.3390

[19] K. Worden, G. Manson, and D. Allman, "Experimental validation of a structural health monitoring methodology: part I. Novelty detection on a laboratory structure," Journal of Sound and Vibration, vol. 259, no. 2, pp. 323-343, 2003. [Online]. Available: https: //doi.org/10.1006/jsvi.2002.5168

[20] G. Manson, K. Worden, and D. Allman, "Experimental validation of a structural health monitoring methodology: part II. Novelty detection on a Gnat aircraft," Journal of Sound and Vibration, vol. 259, no. 2, pp. 345363, 2003. [Online]. Available: https://doi.org/10.1006/jsvi.2002.5167

[21] — , "Experimental validation of a structural health monitoring methodology: part III. Damage location on an aircraft wing," Journal of Sound and Vibration, vol. 259, no. 2, pp. 365-385, 2003. [Online]. Available: https://doi.org/10.1006/jsvi.2002.5169

[22] Y.-L. Zhou, E. Figueiredo, N. Maia, R. Sampaio, and R. Perera, "Damage detection in structures using a transmissibility-based Mahalanobis distance," Structural Control and Health Monitoring, vol. 22, no. 10, pp. 1209-1222, 2015. [Online]. Available: https://doi.org/10.1002/stc.1743

[23] K. Worden, G. Manson, and N. R. J. Fieller, "Damage detection using outlier analysis," Journal of Sound and Vibration, vol. 229, no. 3, pp. 647-667, 2000. [Online]. Available: https://doi.org/10.1006/jsvi.1999. 2514

[24] A. Malhi and R. X. Gao, "PCA-based feature selection scheme for machine defect classification," IEEE Transactions on Instrumentation and Measurement, vol. 53, no. 6, pp. 1517-1525, 2004. [Online]. Available: https://doi.org/10.1109/TIM.2004.834070

[25] R. Shao, W. Hu, Y. Wang, and X. Qi, "The fault feature extraction and classification of gear using principal component analysis and kernel principal component analysis based on the wavelet packet transform," Measurement, vol. 54, pp. 118-132, 2014. [Online]. Available: https://doi.org/10.1016/j.measurement.2014.04.016

[26] J. Xiang, Y. Zhong, and H. Gao, "Rolling element bearing fault detection using PPCA and spectral kurtosis," Measurement, vol. 75, pp. 180-191, 2015. [Online]. Available: https://doi.org/10.1016/j.measurement.2015. 07.045

[27] E. Figueiredo, G. Park, C. R. Farrar, K. Worden, and J. Figueiras, "Machine learning algorithms for damage detection under operational and environmental variability," Structural Health Monitoring, vol. 10, no. 6, pp. 559-572, 2011. [Online]. Available: https://doi.org/10.1177/ 1475921710388971

[28] A. Santos, E. Figueiredo, M. Silva, C. Sales, and J. Costa, "Machine learning algorithms for damage detection: Kernel-based approaches," Journal of Sound and Vibration, vol. 363, pp. 584-599, 2016. [Online]. Available: https://doi.org/10.1016/j.jsv.2015.11.008

[29] N. M. M. Maia, A. P. V. Urgueira, and R. A. B. Almeida, "Whys and wherefores of transmissibility," in Vibration Analysis and Control New Trends and Developments, F. Beltran-Carbajal, Ed. InTech, 2011, pp. 197-216. [Online]. Available: https://doi.org/10.5772/24869

[30] B. Schölkopf, A. Smola, and K.-R. Muller, "Nonlinear component analysis as a kernel eigenvalue problem," Neural Computation, vol. 10, no. 5, pp. 1299-1319, 1998. [Online]. Available: https: //doi.org/10.1162/089976698300017467

[31] B. E. Boser, I. M. Guyon, and V. N. Vapnik, "A training algorithm for optimal margin classifiers," in Proceedings of the Fifth Annual Workshop on Computational Learning Theory. Pittsburgh, Pennsylvania, USA: ACM, Jul 1992, pp. 144-152. [Online]. Available: https://doi.org/10.1145/130385.130401

[32] J. Mercer, "Functions of positive and negative type, and their connection with the theory of integral equations," Philosophical Transactions of the Royal Society of London A: Mathematical, Physical and Engineering Sciences, vol. 209, no. 441-458, pp. 415-446, 1909. [Online]. Available: https://doi.org/10.1098/rsta.1909.0016

[33] S. S. Keerthi and C.-J. Lin, "Asymptotic Behaviors of Support Vector Machines with Gaussian Kernel," Neural Computation, vol. 15, no. 7, pp. 1667-1689, 2003. [Online]. Available: https: //doi.org/10.1162/089976603321891855

[34] D. Widjaja, C. Varon, A. Dorado, J. A. K. Suykens, and S. V. Huffel, "Application of kernel principal component analysis for singlelead-ECG-derived respiration," IEEE Transactions on Biomedical Engineering, vol. 59, no. 4, pp. 1169-1176, 2012. [Online]. Available: https://doi.org/10.1109/TBME.2012.2186448
[35] I. T. Jolliffe, Principal Component Analysis, 2nd ed. New York, United States: Springer-Verlag, 2002, springer Series in Statistics. [Online]. Available: https://doi.org/10.1007/b98835

[36] D. Comaniciu and P. Meer, "Mean shift: a robust approach toward feature space analysis," IEEE Transactions on Pattern Analysis and Machine Intelligence, vol. 24, no. 5, pp. 603-619, May 2002. [Online]. Available: https://doi.org/10.1109/34.1000236

[37] A. Vedaldi and S. Soatto, "Quick shift and kernel methods for mode seeking," in Proceedings of the European Conference on Computer Vision. Springer, Berlin, Heidelberg, 2008, pp. 705-718. [Online]. Available: https://doi.org/10.1007/978-3-540-88693-8_52

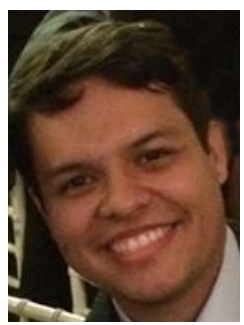

A. Santos received the M.Sc. and Ph.D. degrees in electrical engineering (telecommunications and applied computing) from the Federal Univesity of Pará, Belém, Brazil, in 2014 and 2017, respectively. $\mathrm{He}$ is currently a Professor with the Faculty of Computing and Electrical Engineering, Institute of Geosciences and Engineering, Federal University of Southern and Southeastern Pará (Unifesspa).

His current research interests include machine learning and structural health monitoring.

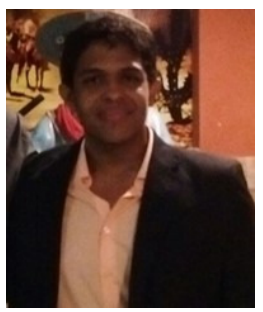

M. F. M. Silva received the B.Sc. degree in computer science and the M.Sc. degree in electrical engineering (applied computing) from the Federal University of Pará, Belém, Brazil, in 2015 and 2017 , respectively, where he is currently pursuing the Ph.D. degree in electrical engineering (applied computing).

His current research interests include machine and deep learning, structural health monitoring, intelligent systems, evolutionary computing, and computer theory.

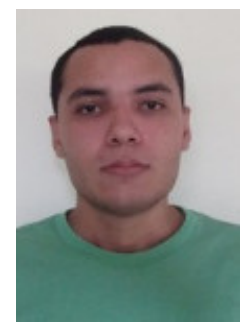

R. Santos received the M.Sc. and Ph.D. degrees in computer science (intelligent systems) from the Federal University of Pará, Belém, Brazil, in 2016 and 2019 , respectively, where he is currently a postdoctoral researcher with Applied Electromagnetism Laboratory.

His current research interests include intelligent systems, evolutionary algorithms, and structural health monitoring.

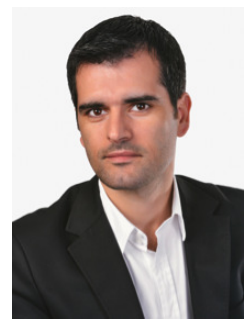

E. Figueiredo received the Ph.D. degree in civil engineering from the Faculty of Engineering of the University of Porto, Porto, Portugal, in 2010.

In 2010, he started teaching at the Catholic University of Portugal, in Lisbon. He is currently Associate Professor in the Faculty of Engineering of the Universidade Lusófona, in Lisbon, and an Integrated Member of Construct - Institute of Research and Development in Structures and Construction, in Porto. His current research interests include structural health monitoring, condition assessment of bridges, structural vibration analysis, finite element modeling, machine learning, and structural damage detection. 


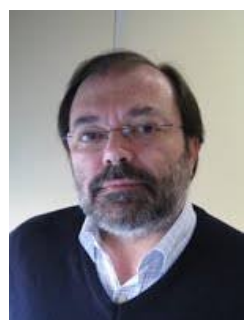

N. M. M. Maia received his habilitation in mechanical engineering in 2001 from Instituto Superior Técnico (IST), University of Lisbon. Prof. Maia has authored and co-authored two textbooks and about 200 scientific publications in international journals and conference proceedings on the subject of modal analysis and structural dynamics.

He obtained his first degree in 1978 and his M.Sc. degree in 1985, both in mechanical engineering from IST, University of Lisbon. He received his Ph.D. in mechanical vibrations (1989) from Imperial College London, UK. His current research interests are modal analysis and modal testing, updating of finite element models, coupling and structural modification, damage detection in structures, modeling of damping, transmissibility in multiple degree-of-freedom systems, and force identification.

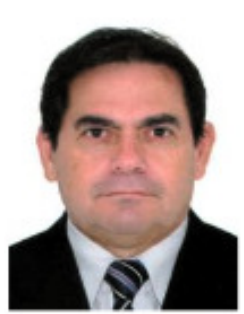

J. C. W. A. Costa (S'94-M'95) received the B.Sc. degree in electrical engineering from the Federal University of Pará (UFPA), Belém, Brazil, in 1981, the M.Sc. degree in electrical engineering from the Pontifical Catholic University of Rio de Janeiro, Rio de Janeiro, Brazil, in 1989, and the Ph.D. degree in electrical engineering from the State University of Campinas, Campinas, Brazil, in 1994

$\mathrm{He}$ is currently a full Professor with the Institute of Technology, UFPA, and a Researcher with the Brazilian Research Funding Agency National Council for Scientific and Technological Development, Brasília, Brazil. His current research interests include broadband systems and optical sensors. 